\title{
Dual-network hydrogel based on ionic nano-reservoir for gastric perforation sealing
}

\author{
Yuehui Yuan ${ }^{1 \dagger}$, Hao $\mathrm{Wu}^{1 \dagger}$, Xueyang Ren ${ }^{1,2 \dagger}$, Jianwu Wang ${ }^{3 \dagger}$, Ruiqing $\mathrm{Liu}^{1}$, Benhui $\mathrm{Hu}^{1,4^{*}}$ and $\mathrm{Ning}^{\mathrm{G}} \mathrm{u}^{1,2^{*}}$
}

\begin{abstract}
In recent years, remarkable progress has been made in the research of injectable hydrogel for internal tissue healing. However, the therapeutic outcome is usually limited when the hydrogel is used for the treatment of gastric perforation due to the high acidic gastric juice and violent deformation of the gastric wall. Regarding these challenges, we proposed an ionic nano-reservoir (INR)-based dual-network hydrogel, which has excellent adhesion and mechanical properties, and can be easily applied to the perforation site to block the perforation while promoting tissue repairing. The results showed that the first network made of polyacrylamide had cross-linked on the stomach tissue within $5 \mathrm{~s}$ under blue light, and enhanced the adhesion performance through mechanical interlock. The nano-hydroxyapatite acted as ionic INR, which can gradually release $\mathrm{Ca}^{2+}$ under acid environments to form the second network with sodium alginate and inhibit the swelling of hydrogel in gastric juice. Meanwhile, the adhesion was further enhanced through amide covalent bonds at the hydrogel-tissue interface with the presence of 1ethyl-3-(3-dimethylaminopropyl)carbodiimide/ $N$-hydroxysuccinimide (EDC/NHS). The dual network hydrogels obtained by the INR strategy could be employed as a potential therapeutic option for gastric perforation and other similar biomedical prolems.
\end{abstract}

Keywords: injectable hydrogel, ionic cross-linking, adhesion, toughness, wound dressing

\section{INTRODUCTION}

Gastric perforation is one of the most severe complications in gastric ulcers and gastric cancer [1]. Moreover, with the advancement of digestive endoscopy, the endoscopic submucosal dissection (ESD) has been widely utilized to treat early gastric cancer, which greatly increases the rate of iatrogenic perforation [2]. The protective mucosal barrier on the gastric wall will be damaged during gastric perforation, which raises the risk of mucosal cell damage by gastric acid, pepsin and bile salt, and further retards self-repair of the gastric wall [3]. Stomach wall perforation may result in the leakage of the acidic gastro- intestinal fluid into the abdominal cavity, causing chemical or bacterial peritonitis, toxic shock and even endangering the patient's life [4]. Simple ulcer perforation and damage by ESD are mainly treated with proton pump inhibitors (PPIs) and mucosal protective agents, while the safety of long-term PPI has been noticed with concern in recent years. There is evidence that long-term PPI use may aggravate small intestine damage and elevate the risk of gastric cancer [5]. For large perforations, surgical treatments, including gastrectomy and simple repair are often used. But surgical treatment also has disadvantages, so that patients' recovery time after surgical treatment is much longer than medication. Laparoscopy has been widely adopted in the treatment of gastric perforation [6]. Despite the economic and safety advantages, the effectiveness of laparoscopy for gastric perforations above $10 \mathrm{~mm}$ in diameter is limited. As a result, more materials or techniques are needed to further improve perforation sealing quality and gastric wall repair using laparoscopic surgery.

Hydrogels have been extensively investigated in tissue engineering and biomedicine due to their advantageous electrical, mechanical, and biological properties [7-21]. Viscous hydrogels, which gel in situ, are characterized by low invasiveness, controllability, and shape adaptability, and are increasingly used for soft tissue repair in vivo [22,23]. Many viscous hydrogels have been developed for bonding dry substrates, while the intermolecular interactions between the hydrogel and wet objects are hindered due to hydration coating [24]. To solve the adhesive issues of hydrogel in wet environments, researchers get inspiration from the adhesive mucus secreted by slugs and have developed a robust adhesive hydrogel that can adhere to wet tissues [25]. In the meanwhile, carboxyl groups in acrylic hydrogels are found to exhibit strong adhesion via hydrogen bond interactions [26]. These hydrogels are mainly designed for the common physiological environment. However, many hydrogels lack the adaptability to the gastric environment because the cross-linked chains are hydrolyzed in an acidic environment [27]. The development of wet-adhesive hydrogels in an acid environment is highly demanded for gastric perforation treatment. The toughness of the hydrogels is another crucial character for their applications in tissue adhesive, espe-

\footnotetext{
${ }^{1}$ School of Biomedical Engineering and Informatics, Nanjing Medical University, Nanjing 211166, China

${ }^{2}$ State Key Laboratory of Bioelectronics, Jiangsu Key Laboratory for Biomaterials and Devices, School of Biological Science and Medical Engineering, Southeast University, Nanjing 210009, China

${ }^{3}$ School of Materials Science and Engineering, Nanyang Technological University, Singapore 639798, Singapore

${ }^{4}$ Jiangsu Key Laboratory of Xenotransplantation, Nanjing Medical University, Nanjing 211166, China

${ }^{\dagger}$ These authors contributed equally to this work.

* Corresponding authors (emails: hubenhui@njmu.edu.cn (Hu B); guning@seu.edu.cn (Gu N))
} 
cially in the stomach, where they are more prone to violent deformation under physiological conditions. In the past decades, numerous tough hydrogels have been developed based on energy dissipation by breaking sacrificial bonds, such as double-network hydrogels [28-30], nanocomposite hydrogels [31-33], ionically coordinated hydrogels [34,35], and dual-crosslinked hydrogels [36-38]. These strategies have their own advantages and can be utilized after adjustment and specific optimization.

As far as we know, the high acidity of gastric acid and violent deformation of gastric wall have been the challenges for gastric perforation treatment. Furthermore, hydrogels are prone to swelling in these situations, leading to the mismatch strain between the hydrogel and the tissue and further affecting the adhesion performance [39]. To solve this problem, we proposed an ionic nano-reservoir (INR)-based dual-network hydrogel strategy, which can be conveniently applied to gastric perforation sites, and maintain long-term adhesion. In this system, the first network of hydrogel polymerized in situ under harmless blue light, which can completely cover the irregularly shaped and severely folded tissue such as the stomach [40]. $\mathrm{Ca}^{2+}$ ions released from the INR (we adopted nano-hydroxyapatite as INR) were complexed to carboxyl groups on the sodium alginate (SA) of the second network of the hydrogel, which greatly enhanced the toughness and inhibited the swelling of hydrogel (Fig. 1a). The hydrogel adhesion was further enhanced through the covalent amide bonds formed between the SA chain of the hydrogel and amine groups of the tissue [41-44]. We tested the feasibility of the hydrogel precursor fluid in rapid gastric ulcer formation in rats and evaluated its therapeutic effect. The obtained hydrogel can adapt to violent deformation and low $\mathrm{pH}$ circumstances, and exhibit stable adhesion and outstanding performances in repairing gastric damage and ulcers.

\section{EXPERIMENTAL SECTION}

\section{Materials}

The following reagents were used: acrylamide (AM, 99\%, Aladdin), N,N'-methylenebisacrylamide (MBAA, 99\%, Aladdin), SA (15-25 cP, Sigma Aldrich), nano-hydroxyapatite (NHA, $\geq 97 \%, \quad<100 \mathrm{~nm}$, Aladdin), lithium phenyl-2,4,6-trimethylbenzoylphosphinate (LAP, $\geq 99.8 \%$, StemEasy), 2-morpholinoethanesulfonic acid monohydrate (MES, $\geq 97 \%$, BioFroxx), 1-ethyl-3-(3-dimethylaminopropyl)carbodiimide (EDC, Aladdin), $N$-hydroxysuccinimide (NHS, Aladdin), paraformaldehyde (PFA, Biosharp), and phosphate-buffered saline (PBS, Bio-Channel). All the reagents were used as received if there is no special statement.

\section{Preparation of INR-based dual-network hydrogel}

The dual-network hydrogel (PAM-SA-NHA, PAM = polyacrylamide) was prepared by the free radical polymerization method [45]. To prepare PAM-SA-NHA hydrogel, SA $(0,1,3$, and $5 \mathrm{wt} \%)$ was dissolved in MES solution at $\mathrm{pH} 6.0$ and stirred overnight to dissolve completely. AM monomer (10, 15, and $20 \mathrm{wt} \%)$ and $\mathrm{NHA}(0,1,2$, and $3 \mathrm{wt} \%)$ were then added. After $10 \mathrm{~min}$ of ultrasonic dispersion, $2 \mathrm{wt} \%$ crosslinker MBAA and $1 \mathrm{wt} \%$ initiator LAP were added into the dispersion to obtain a hydrogel precursor solution. Before gelation, $10 \mathrm{mg} \mathrm{mL}^{-1} \mathrm{EDC} /$ NHS was added to the hydrogel precursor solution and bubbles were removed by ultrasonic, and the gel polymerized under 405-nm blue light for $5 \mathrm{~s}$.

\section{Mechanical tests}

The hydrogel precursor solution was injected into the rectangular Teflon template for curing, and the hydrogel was immersed into MES buffer with different $\mathrm{pH}$ values (1.2, 3.2, 5.2, 7.2) for $2 \mathrm{~h}$. The soaked hydrogel was cut into $40 \mathrm{~mm} \times 20 \mathrm{~mm}$ strips and subjected to tensile testing on a Criterion Electromechanical Test System (C42.503, MTS, loading speed: $20 \mathrm{~mm} \mathrm{~min}^{-1}$ ). The slope of the measured stress-strain curve was the Young's modulus of the hydrogel.

\section{Swelling ratio (SR) characterization}

PAM-SA-NHA hydrogels were soaked in MES buffers of different $\mathrm{pH}$ values until the hydrogel reached the equilibrium state, followed by the measurement of hydrogel weight $\left(W_{s}\right)$. The hydrogel was then lyophilized and the weight of the dry hydrogel $\left(W_{\mathrm{d}}\right)$ was measured. Hydrogel SR was determined with the formula:

$\mathrm{SR}=\frac{W_{\mathrm{s}}-W_{\mathrm{d}}}{W_{\mathrm{d}}}$

\section{Adhesion tests}

Hydrogel adhesion force was characterized with $90^{\circ}$ peeling tests. Pigskin and stomach were purchased from the market and cut into $30 \mathrm{~mm} \times 20 \mathrm{~mm}$ strips. The hydrogel precursor solution was applied to the surface of the tissue, and then coated with pigskin during the gel process to prevent the hydrogel from breaking during the peeling process. After curing, the tissue was incubated in MES buffer solution at different $\mathrm{pH}$ values on an oscillating shaker for $2 \mathrm{~h}$. The tissue was then taken out, and a $90^{\circ}$ peel test was performed at a loading speed of $10 \mathrm{~mm} \mathrm{~min}^{-1}$. Adhesion energy was calculated according to the platform period of the measured force-width ratio [46].

\section{Theoretical calculation of two-dimensional phase diagram between swelling hydrogel and substrate}

Adhesive state simulation was performed between the hydrogel and gastric tissue by calculating two-dimensional phase diagram parameters, where the shear moduli of hydrogel and gastric tissue are defined as $\mu_{\mathrm{f}}$ and $\mu_{\mathrm{s}}$ ( $\mu_{\mathrm{f}}$ was obtained by fitting the measured stress-strain curve of the hydrogel). Hydrogel thickness is defined as $H_{\mathrm{f}}$. Hydrogel adhesion energy $(\Gamma)$ to gastric tissue was calculated according to $90^{\circ}$ peeling tests [39]. The adhesion situation was estimated by fitting calculation results into the two-dimensional phase diagram (the left side of the gray area is delaminated-buckle, and the right side is stable adhesion).

\section{In vitro cytocompatibility of PAM-SA-NHA hydrogel}

The cytocompatibility of hydrogel was verified by live/dead cell staining with human umbilical vein endothelial cells (HUVECs). Hydrogel ( $1 \mathrm{~mm}$ high, $1 \mathrm{~cm}$ wide cylinders) was soaked in $75 \%$ alcohol for $30 \mathrm{~min}$ before cell inoculation and washed with PBS for six times to remove residual alcohol. The hydrogel and cells $\left(300,000 \mathrm{~mL}^{-1}\right)$ were co-cultured in 24 -well plates. The cells were then stained with Calcein-AM and propidium iodide (PI) for 1, 2 , and 3 days and their morphology and proliferation were examined by fluorescence microscopy.

\section{In vitro biocompatibility of PAM-SA-NHA hydrogel}

Inflammatory factors interleukin-6 (IL-6) and tumor necrosis factor- $\alpha$ (TNF- $\alpha$ ) were used to assess the biocompatibility of PAM-SA-NHA hydrogel. After HUVECs were co-cultured with 
hydrogel for $48 \mathrm{~h}$, they were centrifuged at $1000 \times g$ at $4^{\circ} \mathrm{C}$, and the supernatant was collected for IL- 6 and TNF- $\alpha$ measurement by an enzyme linked immunosorbent assay (ELISA) kit.

\section{In vitro blood compatibility of PAM-SA-NHA hydrogel}

Rat caudal vein blood was collected in heparin-treated vials and centrifuged at $1200 \mathrm{r} \mathrm{min}^{-1}$ for $10 \mathrm{~min}$. The supernatant was then removed and washed three times with PBS, and red blood cells were diluted to $5 \%(v / v)$. The hydrogel was homogenized using a tissue grinder and dissolved in PBS at 1, 2, 4 and $8 \mathrm{mg} \mathrm{mL}^{-1}$. The red blood cell suspension and hydrogel dispersion were mixed at a 1:1 volume ratio. PBS was used as negative control and deionized water as positive control. The mixture was then incubated at $37^{\circ} \mathrm{C}$ for $1 \mathrm{~h}$ and then centrifuged at $1200 \mathrm{r} \mathrm{min}^{-1}$ for $10 \mathrm{~min}$. The absorbance of supernatants at $540 \mathrm{~nm}$ was recorded by enzyme calibration.

\section{Therapeutic effect of hydrogel in a rat gastric ulcer model}

Sprague-Dawley rats ( 8 weeks old, $n=6$, half male, half female) were used to establish an acetic acid-induced gastric ulcer model as previously reported [47]. Briefly, the animals were fasted for $72 \mathrm{~h}$ and stomachs isolated under $10 \%$ chloral hydrate anesthesia. A 5-mm-diameter cylindrical PAM hydrogel drenched with acetic acid was adhered on the serosal surface for $90 \mathrm{~s}$ to induce ulcers, and the surface was washed with PBS. The anterior and posterior walls of the stomach were both treated as internal controls. After seven days of regular feeding, the two symmetric ulcers were treated separately, and labelled as the hydrogel treatment and control groups. Precursor mixtures were injected into the ulcer and cured in situ in the treatment group. No treatment was applied to the control group. Stomach samples were collected on day 7 . The ulcerated tissues were then fixed with $4 \%$ PFA for at least $24 \mathrm{~h}$ and embedded in paraffin for histological analysis. All animal experimental protocols and care were approved by the Institutional Animal Care and Use Committee (IACUC-2104054).

\section{RESULTS AND DISCUSSION}

\section{Preparation and characterization of PAM-SA-NHA hydrogels}

The INR-based hydrogel adopted a dual-network structure. The first network is a typical PAM covalent cross-linked network, which was formed via radical polymerization of AM under harmless blue light. The second network was formed by SA and NHA. The uniformly dispersed NHA acts as an INR. With the hydrogel in an acidic environment, the released $\mathrm{Ca}^{2+}$ form coordination bonds with the carboxyl groups on SA, enhancing the crosslinking density on the second network, thereby enhancing hydrogel's toughness in situ (Fig. 1a). The addition of $\mathrm{CaCl}_{2}$ to the solution will directly release a large amount of $\mathrm{Ca}^{2+}$, resulting in the cross-linking of SA, which is not conducive to the use of hydrogel precursors (Fig. S1). The PAM-SA-NHA hydrogel can polymerize within $5 \mathrm{~s}$ under blue light $(405 \mathrm{~nm})$ (Fig. 1b), which simplified the operation. In order to solve the mismatch strain caused by hydrogel swelling, NHA can respond to low $\mathrm{pH}$ of gastric juice, lock the hydrogel molecular chain tightly, and inhibit swelling in gastric juice. Next, the PAM-SANHA hydrogel was immersed in solutions with different $\mathrm{pH}$ values and the swelling was observed. It was found that the diameter of the hydrogel decreased with the reduction of $\mathrm{pH}$ (Fig. 1c). The scanning electron microscopy (SEM) image also showed that the pores of the hydrogel at $\mathrm{pH} 1.2$ (with porosity of $14 \%$ ) were significantly smaller than that at $\mathrm{pH} 7.2$ (with porosity of 33\%) (Fig. 1d).

PAM-SA-NHA hydrogel has excellent tensile properties (stretch rate: 164\%) and can adapt to the large deformation of the stomach during digestion (Fig. 2a). The acid responsiveness is mainly achieved by SA and NHA. Generally, SA chains in

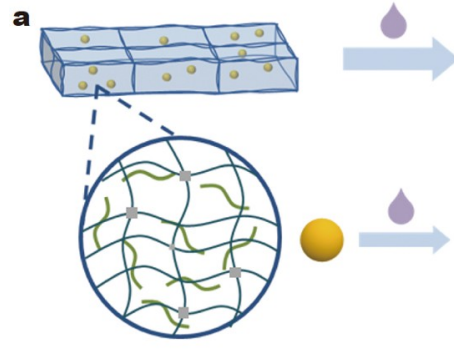

NHA
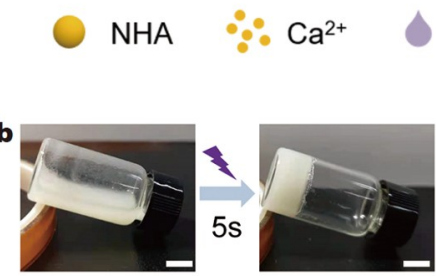

c

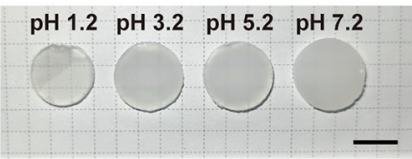

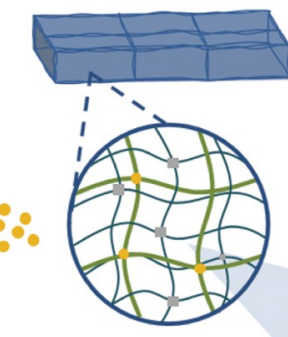

acid

- MBAA

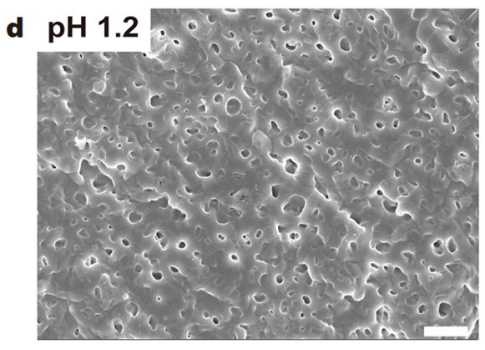

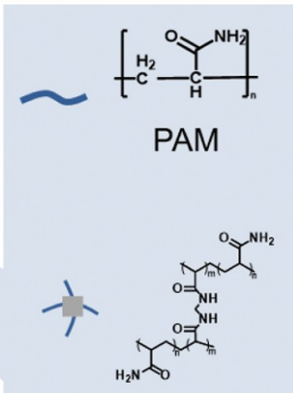

Covalent crosslink

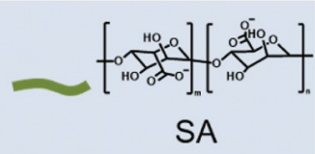

SA

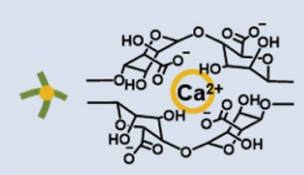

Ionic crosslink

Figure 1 (a) Schematic illustration of in situ fabrication of dual-network hydrogel. (b) Photographs to demonstrate the rapid curing of hydrogel under blue light at $405 \mathrm{~nm}$. Scale bar: $1 \mathrm{~cm}$. (c) Optical images of PAM-SA-NHA hydrogel immersed in MES solution at different pH. Scale bar: $1 \mathrm{~cm}$. (d) SEM images of PAMSA-NHA hydrogels after freeze-drying process (left: treated with $\mathrm{pH} 1.2$ and right: treated with pH 7.2). Scale bar: $5 \mu \mathrm{m}$. 

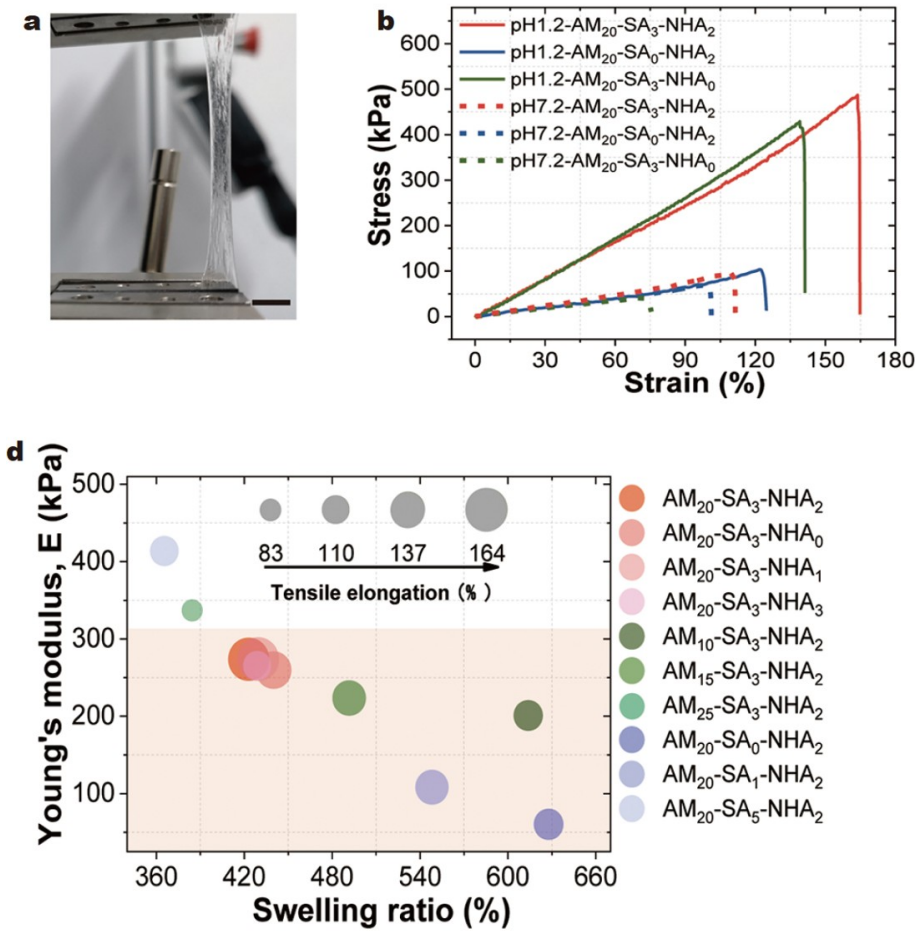
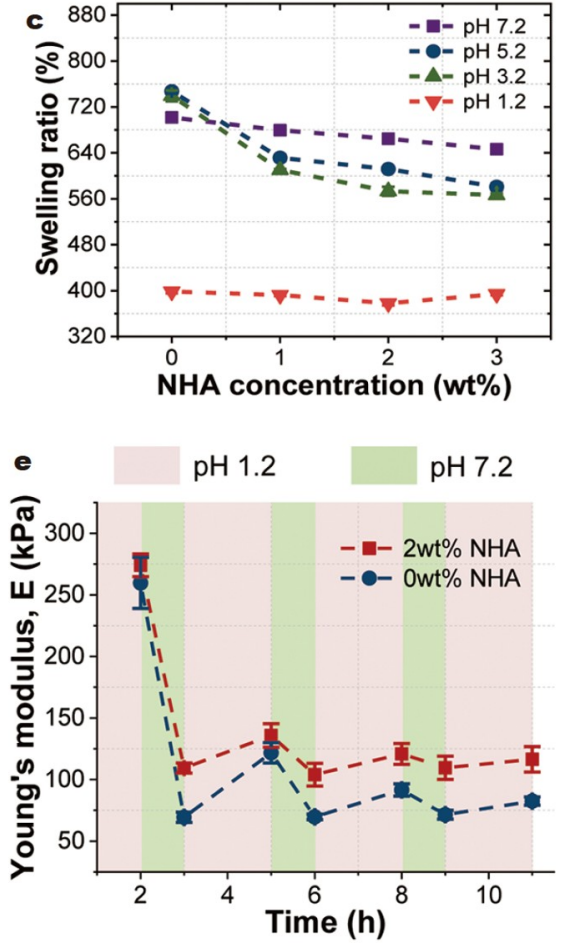

Figure 2 (a) Photograph of the highly stretchable PAM-SA-NHA hydrogel. Scale bar: $1 \mathrm{~cm}$. (b) Tensile stress-strain curves of the PAM-SA-NHA hydrogels at different $\mathrm{pH}$ values with different concentrations of SA and NHA. (c) The SR of PAM-SA-NHA hydrogels at different pH values with different concentrations of NHA. (d) Young's modulus, tensile elongation, and SR of the PAM-SA-NHA hydrogels with different AM, SA, and NHA concentrations. (e) Cyclic change of Young's modulus of the PAM-SA-NHA hydrogels with different concentrations of NHA immersed in MES solution of pH 1.2 (pink) and pH 7.2 (green).

hydrogel may repel each other due to hydrogen ion dissociation in neutral or alkaline conditions, resulting in decreased mechanical properties and high swelling. In an acidic environment, the carboxyl groups on SA are protonated. For this reason, the chains of SA are entangled with each other, thereby increasing the Young's modulus and reducing the SR [48]. Moreover, NHA can react with $\mathrm{H}^{+}$and release $\mathrm{Ca}^{2+}$ to increase the cross-linking point of the $2^{\text {nd }}$ network, thus improving the toughness of hydrogel [49]. We have noticed that redundant NHA can impact the ordered structure of the $2^{\text {nd }}$ network and raise the SR (Fig. 2b, c, Fig. S2). NHA also depleted $\mathrm{H}^{+}$when the hydrogels interacted with stomach acid, which would prevent gastric acid from corroding the wound (Fig. S3). To explore the suitable proportion of the three ingredients, hydrogel's elongation, Young's modulus, and SR were characterized at $\mathrm{pH} 1.2$ and the hydrogel expressed as $\mathrm{AM}_{x}-\mathrm{SA}_{y}-\mathrm{NHA}_{z}(x, y, z$ are the percentages of each component to the total mass). As shown in Fig. 2d, $\mathrm{AM}_{20}-\mathrm{SA}_{3}-\mathrm{NHA}_{2}$ has a higher elongation rate and lower $\mathrm{SR}$, making it more resistive to the large deformation of the stomach and the mismatch strain during swelling. Moreover, the Young's modulus of $\mathrm{AM}_{20}-\mathrm{SA}_{3}-\mathrm{NHA}_{2}$ can also fit well with the stomach tissue $(<316 \mathrm{kPa})$. As a result, the proportion for $\mathrm{AM}_{20^{-}}$ $\mathrm{SA}_{3}-\mathrm{NHA}_{2}$ is optimum for the following experiments. The $\mathrm{pH}$ in the stomach changes frequently, accompanied with severe deformation of the stomach wall. To simulate the internal environment of the stomach, the hydrogel was immersed in MES buffer at $\mathrm{pH} 1.2(4 \mathrm{~h})$ and $\mathrm{pH} 7.2(2 \mathrm{~h})$ alternately [50]. The Young's modulus data were collected by the tensile test with regular intervals, showing that the hydrogel containing NHA has better stability and mechanical properties, confirming the complexation of $\mathrm{Ca}^{2+}$ with SA (Fig. 2e).

\section{Adhesion performance of the hydrogel}

The PAM-SA-NHA hydrogel can stably adhere to tissues via mechanical interlock and chemical bonds. Mechanical interlock was due to the uneven surface of the tissue. When the hydrogel precursor was cast on the tissue, it filled the pores and formed a key-lock topology after polymerization [51,52]. As a result, the friction between hydrogel and tissues was greatly enhanced, where the adhesion energy could reach $108 \mathrm{~J} \mathrm{~m}^{-2}$. Moreover, carboxyl groups in SA sidechains can be activated by EDC/NHS in the hydrogel precursor solution and form amide covalent bonds to enhance the adhesion stability and strength further (Fig. 3a, Fig. S4) [53]. As mentioned above, swelling of hydrogels can result in the mismatch strain between the hydrogel and tissue and further reduce the adhesion energy (Fig. S5). To quantify the adhesion performance of hydrogel in a simulated gastric acid environment, we verified the adhesion energy of hydrogel after immersion in solutions at different $\mathrm{pH}$. We found that the adhesion energy of hydrogel at $\mathrm{pH} 1.2$ was significantly higher than that at $\mathrm{pH} 7.2$ (Fig. 3b, c). The underlying mechanism is that in acidic environments, the hydrogel has lower SR and higher mechanical strength to sustain the peeling force to adhere to the tissues. It was also found that EDC/NHS can significantly improve the adhesion of hydrogel to tissues (Fig. 3b, c, Figs S6 and S7). However, excessive EDC/NHS (over $10 \mathrm{mg} \mathrm{mL}^{-1}$ ) occupied many carboxyl groups, which adversely affected the mechanical properties and adhesion performance 

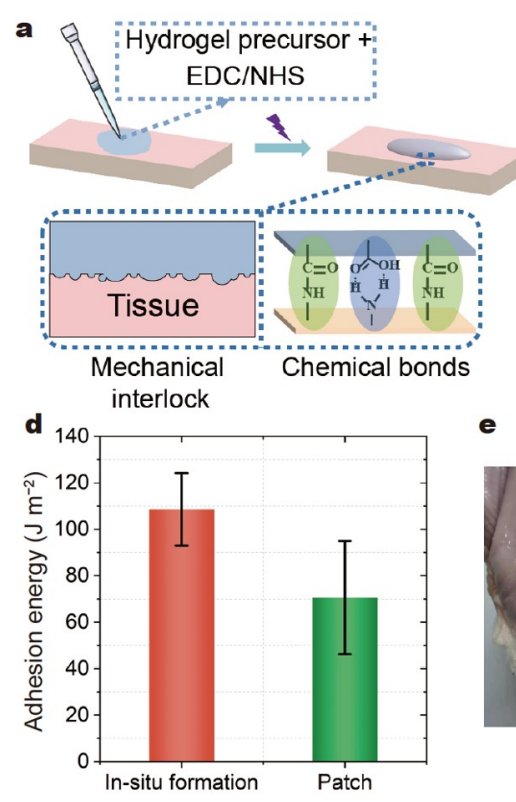
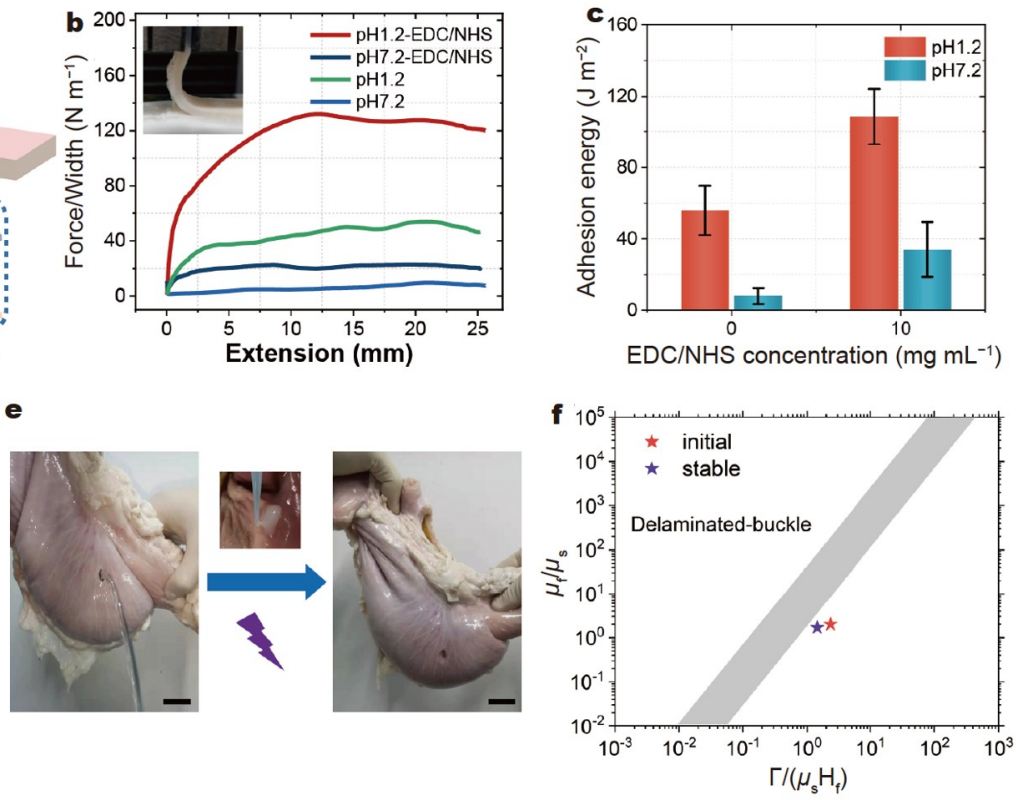

Figure 3 (a) Schematic diagram of the adhesion mechanism of PAM-SA-NHA hydrogel: the formation of adhesion depends on mechanical interlock and chemical bonds (amide bond and hydrogen bond). (b) The impact of EDC/NHS on the force-extension curves of $90^{\circ}$ peeling tests at different pH. (c) Adhesion energy of PAM-SA-NHA hydrogels with or without EDC/NHS in pH 1.2 or 7.2 MES solution, respectively. (d) Comparison of adhesion energy of the in situ formed hydrogel and patched hydrogel. (e) The PAM-SA-NHA hydrogel prevented liquid from flowing out of the injured stomach. Scale bar: $1 \mathrm{~cm}$. (f) The phase diagram of adhesion energy of initial and stable hydrogels (after $12 \mathrm{~h}$ ).

(Fig. S8). To explore the influence of mechanical interlock on hydrogel adhesion properties, a solid hydrogel patch was prepared and used to compare adhesion energy with the hydrogel formed in situ. As shown in Fig. 3d and Fig. S9, the hydrogel formed in situ has greater tissue adhesion than that of solid hydrogel patch, which further supports the effect of mechanical interlock. Hydrogel precursor was used to repair pig stomach perforation to simulate the actual scenarios. After being illuminated by blue light for $5 \mathrm{~s}$, the hydrogel can patch up the perforation site and withstand water pressure from gastric peristalsis without any leakage (Fig. 3e, Figs S10 and S11, and Movie S1). Additionally, the adhesion state between the hydrogel and human stomach showed that the hydrogel adhered to the stomach tissue was calculated by mechanical simulation based on the measurement of parameters including adhesion energy and shear modulus. As shown in the two-dimensional phase diagram (Fig. 3f), the grey band represents the boundary between the delaminated-buckle and unlaminated. The simulated results of the adhesion state between hydrogel and tissue were located in the right of the boundary, indicating that the hydrogel will not fall from the stomach wall at the initial (adhesion for $2 \mathrm{~h}$ ) and after a long period (adhesion for $12 \mathrm{~h}$ ) (Table S1) [54].

\section{Biocompatibility of hydrogel for gastric perforation repair}

The live/dead staining method was used to evaluate the biocompatibility of the hydrogel for gastric perforation repair. To this end, PAM-SA-NHA hydrogel was co-cultured with HUVEC cells. As shown in Fig. 4a and Fig. S12, HUVECs co-cultured with the hydrogel exhibit resemblance morphology and proliferation rate to that in control groups. Considering the implantsin vitro are prone to causing inflammation, TNF- $\alpha$ and
IL-6 levels in the culture media were measured by ELISA. No significant difference was found in the experiment and control groups, which further eliminated the inflammatory risks of PAM-SA-NHA hydrogel (Fig. 4b, c). To evaluate hemocompatibility in vitro, the hydrogel was dispersed in PBS at 1, 2, 4 , and $8 \mathrm{mg} \mathrm{mL}^{-1}$ referred to as $\mathrm{H} 1, \mathrm{H} 2, \mathrm{H} 4$, and $\mathrm{H} 8$, respectively, and incubated with red blood cells for $1 \mathrm{~h}$ at $37^{\circ} \mathrm{C}$ and the supernatant was collected for spectrophotometric analysis [55]. PBS and deionized water were used as negative and positive controls, respectively. As shown in Fig. 4d, e, no significant difference was found among the hydrogel-treated groups and the negative control group; blood cells were precipitated in the centrifuges, and the supernatant had a low level of optical density (OD). In contrast, the blood cells in positive controls were lysed and the supernatant appeared bright red and exhibited significantly greater OD value relative to the other groups, indicating that the hydrogel has good blood compatibility.

\section{The repairing effect of PAM-SA-NHA hydrogel on rats' gastric ulcer model}

To evaluate the repair capabilities of PAM-SA-NHA hydrogel on gastric ulcer injury, the acetic acid-induced rat models of gastric ulcer were established (both sides of the tissue with the size of $\sim 1.4 \mathrm{~mm}^{2}, n=6$ ). PAM-SA-NHA hydrogel was applied to one side of the gastric ulcer, while another size was left untreated as control. Seven days after surgery, the gastric ulcer was obviously healed, exhibiting much smaller perforation than that in control sites ( $95 \%$ reduction), while the marked necrosis was observable in control stomach tissue (Fig. 5a). Haematoxylin and eosin $(\mathrm{H} \& \mathrm{E})$ staining analysis was also carried out to investigate the recovery status of gastric ulcers. As shown in Fig. 5b, massive parenchymal cell necrosis on the mucosal layer at the control 


\section{Live/Dead Assay}
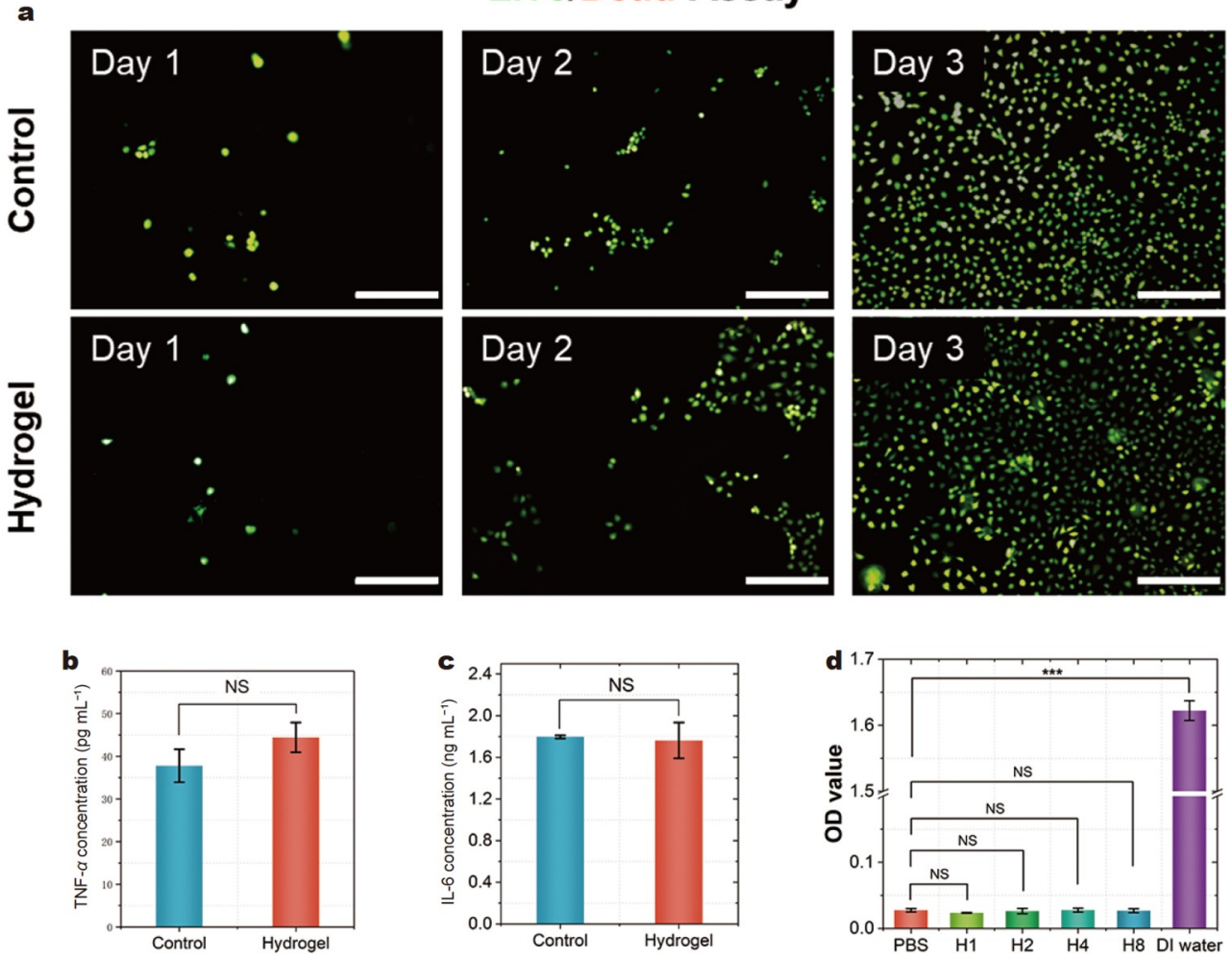

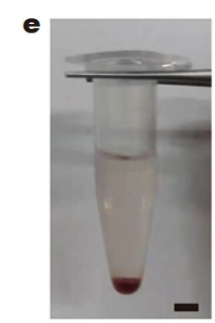

PBS

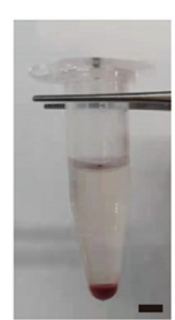

$\mathrm{H} 1$

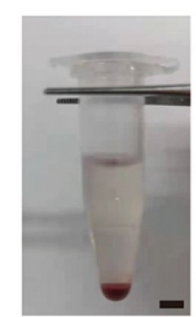

$\mathrm{H} 2$

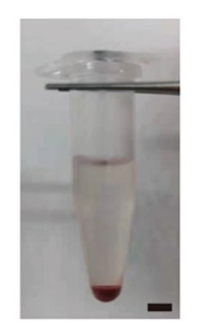

$\mathrm{H} 4$

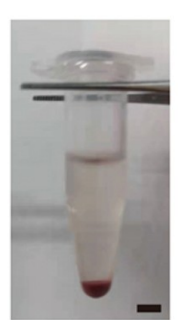

$\mathrm{H} 8$

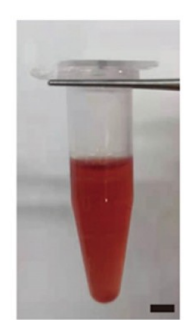

DI water

Figure 4 (a) Live/dead staining of HUVEC cells after being treated with or without PAM-SA-NHA hydrogels for different times of incubation. Scale bar: $100 \mu \mathrm{m}$. (b, c) Quantitative detection of cell inflammatory factor concentration by ELISA (NS, not significant; $n=3$ ). (d) OD values of blood supernatant of different groups $\left({ }^{* * *} p<0.0001, n=3\right)$. (e) Photographs of blood treated with different groups. Scale bar: $5 \mathrm{~mm}$.

site is considerably higher than that at the hydrogel treatment site. Moreover, granulation tissue proliferation was observed in muscularis, while severe hemorrhage and submucosa edema with proliferation of granulation tissue in lamina propria were observed in the control group. Therefore, the PAM-SA-NHA hydrogel has alleviated the damage development caused by acetic acid (Fig. 5b, Fig. S13). To evaluate the effect of PAM-SANHA hydrogel on the healing of rat gastric ulcers, immunostaining was carried out to measure the expression of CD31 (a vascular endothelial cell marker) and proliferating cell nuclear antigen (PCNA, an indicator of cell proliferation) in the hydrogel-treated site and control site. These factors indicate angiogenesis and re-epithelialization, respectively. As shown in Fig. 5c-e, significantly higher CD31 staining intensity (maroon) was observed at the hydrogel-treated site than that at the control site $(p<0.01)$, indicating that the hydrogel enhanced the submucosa capillary density at the ulcer and promoted ulcer healing. Similarly, PCNA staining intensity (maroon) in the hydrogel-treated site was significantly higher relative to the control site $(p<0.05)$, indicating enhanced cell proliferation. In conclusion, our data indicated that the PAM-SA-NHA hydrogel could supress inflammation, promote vascular regeneration, accelerate cell proliferation, and finally enhance the gastric ulcer healing process.

\section{CONCLUSIONS}

In summary, we have designed a dual-network hydrogel that exhibited advantageous properties to perforation sealing during gastric ulcer injury. The NHA in the hydrogel can consume $\mathrm{H}^{+}$ in the gastric acids, and release $\mathrm{Ca}^{2+}$ further to improve the mechanical and adhesion properties of hydrogel. After being applied to the gastric wall, the PAM-SA-NHA hydrogel can act as a protective barrier to hinder the penetration of catabolic factors in gastric juice, inhibit inflammation, and enhance vascular regeneration and cell proliferation, and finally promote ulcer healing. The responsive release INR has exhibited pro- 

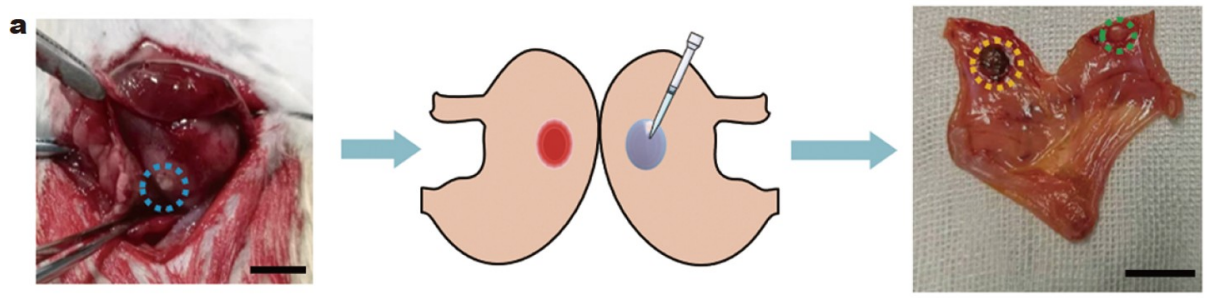

. . . . . . . . . Gastric ulcer

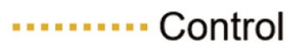

Hydrogel

b
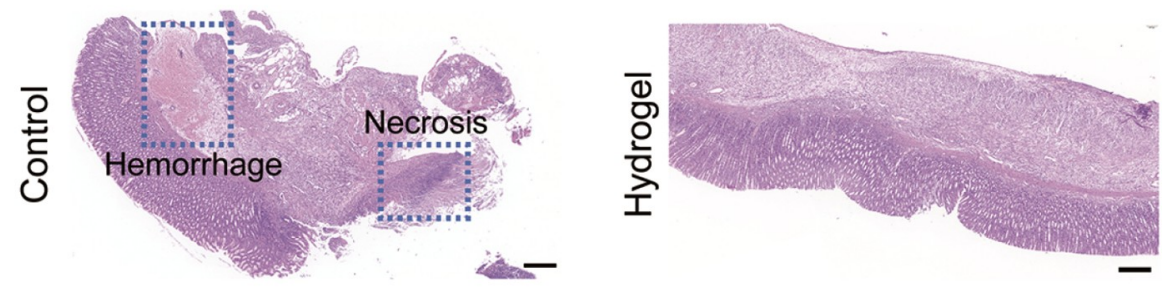

CD31

\section{c}
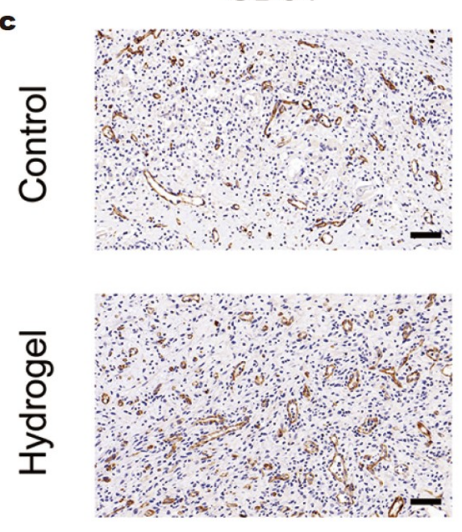
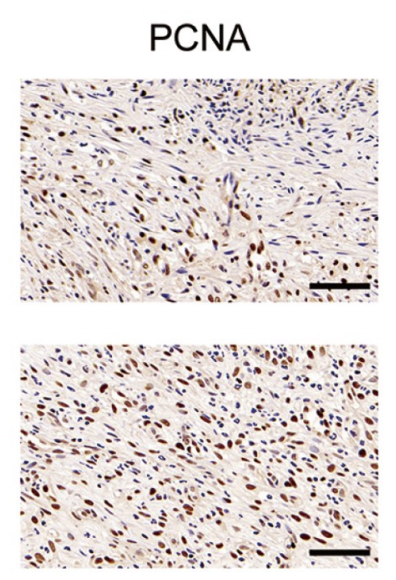

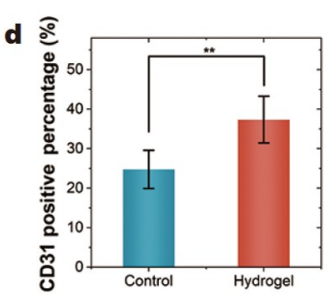

.

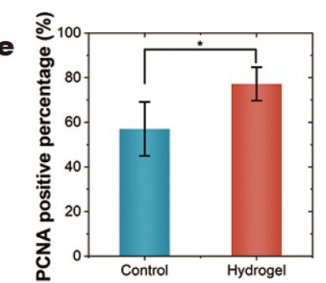

Figure 5 (a) Photographs of acetic acid-induced gastric ulcers in rats, and treatment effects of PAM-SA-NHA hydrogels compared with control groups after 7 days. Scale bar: $1 \mathrm{~cm}$. (b) H\&E staining of gastric ulcers treated with or without PAM-SA-NHA hydrogels. Scale bar: $300 \mu \mathrm{m}$. (c) Immunohistochemistry staining for CD31 and PCNA of gastric ulcers treated with or without PAM-SA-NHA hydrogels. Scale bar: $150 \mu \mathrm{m}$. (d) Quantification of the relative area of CD31 positive capillaries. (e) Quantitative analysis of the proportion of PCNA-positive cells $\left({ }^{*} p<0.05,{ }^{* *} p<0.01\right.$, and $n=6$ ).

mising advantages in adjusting the mechanical properties and other environmental suitability, which can be applied in other in situ applications.

Received 28 September 2021; accepted 27 October 2021; published online 15 December 2021

1 Weledji EP. An overview of gastroduodenal perforation. Front Surg, 2020, 7: 573901

2 Xu X, Xia X, Zhang $\mathrm{K}$, et al. Bioadhesive hydrogels demonstrating $\mathrm{pH}$ independent and ultrafast gelation promote gastric ulcer healing in pigs. Sci Transl Med, 2020, 12: eaba8014

3 Linden SK, Sutton P, Karlsson NG, et al. Mucins in the mucosal barrier to infection. Mucosal Immunol, 2008, 1: 183-197

4 Peery AF, Dellon ES, Lund J, et al. Burden of gastrointestinal disease in the United States: 2012 update. Gastroenterology, 2012, 143: 11791187.e3

5 Cheung KS, Chan EW, Wong AYS, et al. Long-term proton pump inhibitors and risk of gastric cancer development after treatment for Helicobacter pylori: A population-based study. Gut, 2018, 67: 28-35

6 Kim HS, Lee JH, Kim MG. Kim H S, Lee J H. Outcomes of laparoscopic primary gastrectomy with curative intent for gastric perforation: Ex- perience from a single surgeon. Surg Endosc, 2021, 35: 4206-4213

7 Xue K, Wang X, Yong PW, et al. Hydrogels as emerging materials for translational biomedicine. Adv Therap, 2019, 2: 1800088

8 Kharaziha M, Baidya A, Annabi N. Rational design of immunomodulatory hydrogels for chronic wound healing. Adv Mater, 2021, 33: 2100176

9 Yin X, Hao Y, Lu Y, et al. Bio-multifunctional hydrogel patches for repairing full-thickness abdominal wall defects. Adv Funct Mater, 2021, 31: 2105614

10 Peng X, Xia X, Xu X, et al. Ultrafast self-gelling powder mediates robust wet adhesion to promote healing of gastrointestinal perforations. Sci Adv, 2021, 7: eabe8739

11 Gao Y, Han X, Chen J, et al. Hydrogel-mesh composite for wound closure. Proc Natl Acad Sci USA, 2021, 118: e2103457118

12 Li S, Cong Y, Fu J. Tissue adhesive hydrogel bioelectronics. J Mater Chem B, 2021, 9: 4423-4443

13 Zhou L, Dai C, Fan L, et al. Injectable self-healing natural biopolymerbased hydrogel adhesive with thermoresponsive reversible adhesion for minimally invasive surgery. Adv Funct Mater, 2021, 31: 2007457

14 Yuk H, Lu B, Zhao X. Hydrogel bioelectronics. Chem Soc Rev, 2019, 48: 1642-1667

15 Lin J, Wang Z, Huang J, et al. Microenvironment-protected exosomehydrogel for facilitating endometrial regeneration, fertility restoration, 
and live birth of offspring. Small, 2021, 17: 2007235

16 Wang $\mathrm{P}, \mathrm{Pu} \mathrm{Y}$, Ren Y, et al. Bio-inspired hydrogel-based bandage with robust adhesive and antibacterial abilities for skin closure. Sci China Mater, 2021, : doi: 10.1007/s40843-021-1724-8

$17 \mathrm{Ma} \mathrm{H}, \mathrm{He} \mathrm{C}$, Chen X. Ma H, He C. Injectable hydrogels as local depots at tumor sites for antitumor immunotherapy and immune-based combination therapy. Macromol Biosci, 2021, 21: 2100039

18 Pei X, Zhang H, Zhou Y, et al. Stretchable, self-healing and tissueadhesive zwitterionic hydrogels as strain sensors for wireless monitoring of organ motions. Mater Horiz, 2020, 7: 1872-1882

19 Tang W, Zhou Y, Chen S, et al. Delamination-resistant imperceptible bioelectrode for robust electrophysiological signals monitoring. ACS Mater Lett, 2021, 3: 1385-1393

$20 \mathrm{Hu}$ B, Berkey C, Feliciano T, et al. Thermal-disrupting interface mitigates intercellular cohesion loss for accurate topical antibacterial therapy. Adv Mater, 2020, 32: 1907030

21 Wang Z, Chen J, Wang L, et al. Flexible and wearable strain sensors based on tough and self-adhesive ion conducting hydrogels. J Mater Chem B, 2019, 7: 24-29

22 Lee HP, Lokhande G, Singh KA, et al. Light-triggered in situ gelation of hydrogels using $2 \mathrm{D}$ molybdenum disulfide $\left(\mathrm{MoS}_{2}\right)$ nanoassemblies as crosslink epicenter. Adv Mater, 2021, 33: 2101238

23 Zhang $\mathrm{Z}$, $\mathrm{He} \mathrm{C}$, Rong $\mathrm{Y}$, et al. A fast and versatile cross-linking strategy via $o$-phthalaldehyde condensation for mechanically strengthened and functional hydrogels. Natl Sci Rev, 2021, 8: nwaa128

24 Xie $\mathrm{C}$, Wang X, He $\mathrm{H}$, et al. Mussel-inspired hydrogels for self-adhesive bioelectronics. Adv Funct Mater, 2020, 30: 1909954

25 Li J, Celiz AD, Yang J, et al. Tough adhesives for diverse wet surfaces. Science, 2017, 357: 378-381

26 Gan $\mathrm{D}, \mathrm{Xu} \mathrm{T}$, Xing $\mathrm{W}$, et al. Mussel-inspired contact-active antibacterial hydrogel with high cell affinity, toughness, and recoverability. Adv Funct Mater, 2019, 29: 1805964

27 Chen Y, Tan Z, Wang W, et al. Injectable, self-healing, and multiresponsive hydrogels via dynamic covalent bond formation between benzoxaborole and hydroxyl groups. Biomacromolecules, 2018, 20: 1028-1035

28 Gong JP, Katsuyama Y, Kurokawa T, et al. Double-network hydrogels with extremely high mechanical strength. Adv Mater, 2003, 15: 11551158

29 Matsuda T, Kawakami R, Namba R, et al. Mechanoresponsive selfgrowing hydrogels inspired by muscle training. Science, 2019, 363: 504508

30 Pourjavadi A, Tavakolizadeh M, Hosseini SH, et al. Highly stretchable, self-adhesive, and self-healable double network hydrogel based on alginate/polyacrylamide with tunable mechanical properties. J Polym Sci, 2020, 58: 2062-2073

31 Liu Z, Faraj Y, Ju XJ, et al. Nanocomposite smart hydrogels with improved responsiveness and mechanical properties: A mini review. J Polym Sci Part B-Polym Phys, 2018, 56: 1306-1313

32 Ding J, Chen P, Wang B, et al. Rational design of coplanar polypyrrolebased graphene hydrogels with excellent energy-storage performance. Small Struct, 2021, 2: 2100073

33 Zhou Y, Liu L, Cao Y, et al. A nanocomposite vehicle based on metalorganic framework nanoparticle incorporated biodegradable microspheres for enhanced oral insulin delivery. ACS Appl Mater Interfaces, 2020, 12: 22581-22592

34 Sun JY, Zhao X, Illeperuma WRK, et al. Highly stretchable and tough hydrogels. Nature, 2012, 489: 133-136

35 Bai J, Wang R, Ju M, et al. Facile preparation and high performance of wearable strain sensors based on ionically cross-linked composite hydrogels. Sci China Mater, 2021, 64: 942-952

36 Wang YJ, Zhang XN, Song Y, et al. Ultrastiff and tough supramolecular hydrogels with a dense and robust hydrogen bond network. Chem Mater, 2019, 31: 1430-1440

37 Mo F, Wang Z, Jiang R, et al. Energy-dissipative dual-crosslinked hydrogels for dynamically super-tough sensors. Sci China Mater, 2021, 64: 2764-2776

38 Kim ES, Song DB, Choi KH, et al. Robust and recoverable dual crosslinking networks in pressure-sensitive adhesives. J Polym Sci, 2020, 58

\section{8-3369}

39 Wang Q, Zhao X. A three-dimensional phase diagram of growthinduced surface instabilities. Sci Rep, 2015, 5: 8887

40 Guo Q, Sun $\mathrm{H}, \mathrm{Wu} \mathrm{X}$, et al. In situ clickable purely zwitterionic hydrogel for peritoneal adhesion prevention. Chem Mater, 2020, 32: 6347-6357

41 Chen $\mathrm{X}$, Yuk $\mathrm{H}, \mathrm{Wu}$ J, et al. Instant tough bioadhesive with triggerable benign detachment. Proc Natl Acad Sci USA, 2020, 117: 15497-15503

42 Yuk H, Varela CE, Nabzdyk CS, et al. Dry double-sided tape for adhesion of wet tissues and devices. Nature, 2019, 575: 169-174

43 Deng J, Yuk $\mathrm{H}, \mathrm{Wu}$ J, et al. Electrical bioadhesive interface for bioelectronics. Nat Mater, 2021, 20: 229-236

44 Pei X, Wang J, Cong Y, et al. Recent progress in polymer hydrogel bioadhesives. J Polym Sci, 2021, 59: 1312-1337

45 Zhang G, Chen S, Peng Z, et al. Topologically enhanced dual-network hydrogels with rapid recovery for low-hysteresis, self-adhesive epidemic electronics. ACS Appl Mater Interfaces, 2021, 13: 12531-12540

46 Yuk H, Zhang T, Lin S, et al. Tough bonding of hydrogels to diverse non-porous surfaces. Nat Mater, 2016, 15: 190-196

47 Okabe S, Amagase K. An overview of acetic acid ulcer models: The history and state of the art of peptic ulcer research. Biol Pharm Bull, 2005, 28: 1321-1341

48 Wang W, Wang A. Synthesis and swelling properties of $\mathrm{pH}$-sensitive semi-IPN superabsorbent hydrogels based on sodium alginate-g-poly (sodium acrylate) and polyvinylpyrrolidone. Carbohyd Polym, 2010, 80: 1028-1036

49 Christoffersen J, Christoffersen MR, Arends J, et al. Formation of phosphate-containing calcium fluoride at the expense of enamel, hydroxyapatite and fluorapatite. Caries Res, 1995, 29: 223-230

50 Ovesen L, Bendtsen F, Tage-Jensen U, et al. Intraluminal $\mathrm{pH}$ in the stomach, duodenum, and proximal jejunum in normal subjects and patients with exocrine pancreatic insufficiency. Gastroenterology, 1986, 90: 958-962

51 Yang J, Bai R, Chen B, et al. Hydrogel adhesion: A supramolecular synergy of chemistry, topology, and mechanics. Adv Funct Mater, 2020, 30: 1901693

52 Zhu M, Zhang F, Chen X. Bioinspired mechanically interlocking structures. Small Struct, 2020, 1: 2000045

53 Wang C, Yan Q, Liu HB, et al. Different EDC/NHS activation mechanisms between PAA and PMAA brushes and the following amidation reactions. Langmuir, 2011, 27: 12058-12068

54 Saraf H, Ramesh KT, Lennon AM, et al. Mechanical properties of soft human tissues under dynamic loading. J BioMech, 2007, 40: 1960-1967

55 Huang W, Cheng S, Wang X, et al. Noncompressible hemostasis and bone regeneration induced by an absorbable bioadhesive self-healing hydrogel. Adv Funct Mater, 2021, 31: 2009189

Acknowledgements This work was supported by the National Natural Science Foundation of China (81971701, 51832001, and 81901873), the Natural Science Foundation of Jiangsu Province (BK20201352), and the Program of Jiangsu Specially-Appointed Professor.

Author contributions Yuan $\mathrm{Y}, \mathrm{Wu} \mathrm{H}, \mathrm{Wang} \mathrm{J}$, and Ren $\mathrm{X}$ designed and performed the experiments, and wrote the paper; Liu R completed the data curation; $\mathrm{Gu} \mathrm{N}$ and Hu B proposed the concept and supervised this study. All authors contributed to the general discussion and revision of the manuscript.

Conflict of interest The authors declare that they have no conflict of interest.

Supplementary information Supporting data are available in the online version of the paper. 


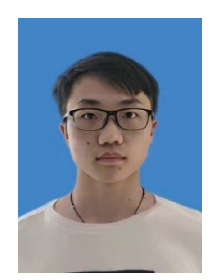

Yuehui Yuan is studying for his Master's degree at the School of Biomedical Engineering and Informatics, Nanjing Medical University (NJMU). His current research interest is the flexible diagnosis and treatment integrated materials.

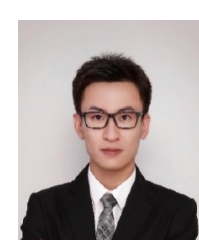

Benhui $\mathrm{Hu}$ received his $\mathrm{PhD}$ degree from Nanyang Technological University in Singapore. He started an independent research career as a full professor at the School of Biomedical Engineering and Informatics, NJMU in 2018, and served as the deputy director of the Department of Biomedical Engineering, NJMU. His research focuses on implantable/wearable diagnostic and therapeutic flexible devices, which are used in real-time multimodal monitoring and boosted tissue repair.

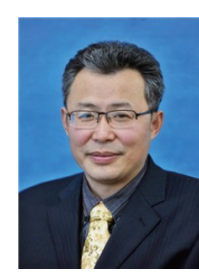

Ning Gu was born in 1964. He received his PhD degree in biomedical engineering from the Department of Biomedical Engineering, Southeast University, Nanjing, China, in 1996. Currently, he is a Changjiang Scholar Professor and NSFC Outstanding Young Investigator Fund Winner at the School of Biological Science and Medical Engineering, Southeast University. He also serves as the director of Jiangsu Key Laboratory for Biomaterials and Devices, the president of Jiangsu Society of Biomedical Engineering, the director of the Research Center for $\mathrm{Na}$ noscale Science and Technology, Southeast University. His research interests include biomaterials, nanobiology, medical imaging, and advanced instrument development.

\section{基于纳米离子储层的双网络胃穿孔封堵水凝胶}

袁月辉 ${ }^{1 \dagger}$, 吴吴 ${ }^{1 \dagger}$, 任雪洋 ${ }^{1,2 \dagger}$, 王建武 ${ }^{\dagger \dagger}$, 刘瑞清 ${ }^{1}$, 胡本慧 $1,4^{*}$, 顾宁 ${ }^{1,2^{*}}$

摘要 近年来, 对于可注射水凝胶用于内部组织损伤修复的研究取得 了一定进展, 但将此类水凝胶用于胃穿孔治疗时, 依然面临强酸性胃液 及胃壁的剧烈形变带来的严峻问题. 鉴于此, 我们提出了一种基于纳米 离子储库(INR)的双网络水凝胶策略, 该水凝胶具有优异的黏附和机械 性能, 可以简便地涂覆于穿孔部位，在封堵穿孔的同时促进组织修复. 结果显示, 在蓝光条件下, 聚丙烯酰胺 (PAM) 可以于 $5 \mathrm{~s}$ 内在胃组织上交 联形成第一重网络, 并通过机械互锁增强黏附性能; 此外, 纳米差基磷 灰石作为纳米离子储库可在酸性环境下释放 $\mathrm{Ca}^{2+}$, 与海藻酸钠 $(\mathrm{SA})$ 形成 第二重网络, 抑制水凝胶在胃液中的溶胀. 同时, 通过在体系中引入碳 二亚胺 $/ N$-差基琥珀酰亚胺(EDC/NHS), 可以促进水凝胶-组织界面上 酯酰胺共价键的形成, 从而进一步增强黏附力. 因此我们认为, 这种通 过纳米离子储库策略获得的双网络水凝胶有望用于解决胃穿孔乃至其 他类似的生物医学问题. 